\title{
Cardiac interventional procedures in the UK 1992 to 1996
}

\author{
H H Gray, on behalf of the Council of the British Cardiovascular Intervention Society
}

In the UK most patients undergoing diagnostic catheterisation and interventional cardiac procedures are treated in National Health Service (NHS) hospitals. However, a number of private hospitals also undertake such procedures and are reimbursed through other mechanisms such as private health insurance, overseas contracts, or patient self funding, and some of these patients are foreign nationals. Also, a small number of privately funded patients have their procedures undertaken in NHS hospitals, and an even smaller number of NHS patients have their procedures contracted to the private sector. These variations make it difficult to make accurate assessments of national activities per head of the UK population. Nevertheless, around $90 \%$ of all interventional procedures are undertaken in NHS hospitals and over $95 \%$ of these patients are UK citizens being treated under the NHS. This allows us to make a reasonable estimate of national activity with regard to coronary intervention.

Over the last decade there has been a considerable expansion in the number of diagnostic catheter procedures being undertaken in laboratories situated in district general hospitals, and there has been an expansion in the number of consultant cardiologists. More recently, mobile laboratories have also been used by a number of district hospitals. In contrast, the number of centres undertaking interventional procedures has changed very little; this may reflect the ability of existing centres to increase the volume of procedures undertaken and concerns about the need for sites to have on site cardiac surgery.

The interventional procedures reported here refer to the total number of procedures undertaken. Since some patients will undergo more than one procedure in a single calendar year, the number of interventions does not equate with the number of patients treated.

Council of the Briovascular
Cardiovation Intervention Society E J Perrins (president) M Norell (secretary) P Silverton (treasurer)

H H Gray (audit secretary)

$M$ de Belder

R Bosley

S W Davies

K D Dawkins

A Gershlick

R J C Hall

D Ramsdale

P Schofield

L D R Smith

Correspondence to: Dr H H Gray, Wessex Cardiac Unit, Southampton University Hospital,

Southampton SO16 6YD, UK. Methods

All centres in the UK (England, Wales, Scotland, and Northern Ireland) undertaking percutaneous interventional cardiac procedures have returned data on these procedures to the British Cardiovascular Intervention Society since 1988, and these have been the subject of previous reports. ${ }^{1-4}$ This report covers activity in the UK over the years 1992 to 1996 and relates only to procedures on adults and not to those involving children. The definitions used in this report of outcome measures, and the clinical settings in which angioplasty procedures were undertaken, are the same as those given in the 1989 report. $^{2}$

In 1992 a total of 42 NHS centres and 10 private centres undertook coronary angio- plasty; these figures changed to 41 and 12, respectively, in 1996. All these centres were approached to provide data relating to their interventional and diagnostic procedures. For interventional procedures data were therefore sought from all centres undertaking such procedures in the UK at the time. However, a large number of diagnostic procedures are undertaken at non-interventional centres, and these hospitals were not surveyed, so data concerning diagnostic procedures is from a subset of the total number undertaken nationally. The total number of coronary arteriograms undertaken to generate the percutaneous transluminal coronary angioplasty (PTCA) workload in the UK reported below is therefore uncertain.

Questionnaires were sent to each centre requesting information relating to facilities, medical staffing, and the number of diagnostic and interventional procedures. The principal purpose of the survey was to determine levels of activity and trends over the five year period, but centres were also asked to give information concerning outcome following PTCA. Methods of data collection varied enormously between centres. Most were able to report major complications occurring within the catheter laboratory during an interventional procedure and soon after the procedure, such as death and requirement for emergency coronary artery bypass grafting (CABG), but data on subsequent myocardial infarction or other in-hospital complications were less frequently reported by centres and data that were returned are likely to be underestimates of the true total. For the collection of post-procedural data most centres rely on retrospective analysis of case notes, which is time consuming and may not be comprehensive. Hence, some infarcts may have been missed. The questionnaire related only to in-hospital stay and no subsequent follow up data were requested.

The process of data collection within centres

was rarely funded separately and usually relied on the enthusiasm and diligence of volunteers from various staff groups such as nurses, physiological measurement technicians, radiographers, and medical and secretarial staff. The majority of centres returned most of the requested data and the number of procedures from which mean results were derived was therefore large. As such, useful information can be derived. However, the outcome of procedures in those centres not returning data obviously cannot be known, and the case mix in these centres may be different from that in the centres returning data. Hence, a degree of caution is required before extrapolations are made from the means of returned data to create "national" averages against which local 
Table 1 Number of catheter laboratories, volume of procedures, and staffing levels in NHS centres surveyed

\begin{tabular}{lrrrrr}
\hline & 1992 & 1993 & 1994 & 1995 & 1996 \\
\hline Mean number of catheter laboratories per centre & 1.7 & 1.7 & 1.8 & 1.8 & 1.9 \\
Mean number of adult catheter sessions per week & 12.5 & 13.1 & 15.0 & 15.5 & 16.1 \\
$\begin{array}{l}\text { Mean annual number of adult diagnostic angiograms per centre } \\
\text { Mean annual number of adult pacemaker procedures per centre }\end{array}$ & 1593 & 1657 & 1840 & 2051 & 2087 \\
$\begin{array}{l}\text { Mean annual number of adult intervention procedures per centre } \\
\text { Mean number of consultant cardiologists undertaking diagnostic }\end{array}$ & 243 & 226 & 239 & 251 & 336 \\
$\quad$ catheterisation per centre & 284 & 321 & 373 & 460 \\
$\begin{array}{l}\text { Mean number of junior cardiology staff undertaking diagnostic } \\
\quad \text { catheterisation per centre }\end{array}$ & 4.8 & 5.2 & 5.7 & 6.7 & 7.6 \\
$\begin{array}{l}\text { Mean number of radiologists (consultant and junior) undertaking } \\
\quad \text { diagnostic catheterisation per centre }\end{array}$ & 3.9 & 4.1 & 4.6 & 5.3 & 6.3 \\
$\begin{array}{l}\text { Mean number of trained PTCA operators (cardiologists) per centre } \\
\text { Mean number of trained PTCA operators (radiologists) per centre }\end{array}$ & 0.7 & 0.9 & 0.8 & 1.2 & 0.7 \\
\hline
\end{tabular}

performance might be compared. Risk stratified outcome following cardiac interventional procedures is currently being audited in the pilot centres participating in the UK central cardiac audit database project.

\section{Results}

All centres undertaking adult interventional cardiac procedures were asked to supply information regarding the overall adult workload of their cardiac catheter laboratories and the operators undertaking this work. Since the volume of procedures and pattern of work is different between NHS and private centres, their data have been separated (NHS centres in table 1 and private centres in table 2).

\section{CATHETER LABORATORIES}

In NHS interventional centres the mean number of catheter laboratories rose from 1.7 per centre in 1992 to 1.9 in 1996 (table 1). Defining a single session of catheter laboratory time as equal to half a normal working day, a single catheter laboratory has the potential for a total of 10 sessions to be commissioned in a normal five day working week. Between 1992 and 1996 there was a reported rise in the mean number of sessions commissioned weekly for adult work from 12.5 to 16.1 , respectively. Allowing for the additional unreported sessions allocated to electrophysiology and to paediatric work in those centres undertaking paediatric procedures, it is likely that most centres have commissioned all of their potential catheter laboratory sessions.

In contrast there was no consistent change in either the mean number of catheter laboratories or of commissioned sessions in the private sector (table 2). However, the number of private centres providing this information was small and private hospitals often undertake routine catheter laboratory work outside normal working hours, making direct comparison with the NHS more difficult.

VOLUME OF PROCEDURES

The number of diagnostic angiograms, pacemakers, and interventional procedures in NHS centres all increased in the five year period, from a mean annual grand total of 2024 per centre in 1992 to 2883 in 1996, an increase of $42 \%$ (table 1). This gives a mean for the annual grand total of procedures undertaken per catheter laboratory of 1191 in 1992 and 1517 in 1996, and per annual catheter laboratory session of 162 and 179 , respectively. Given the increasing complexity of the procedures undertaken over these five years, this suggests an increase in efficiency and speed of throughput, though possibly also reflects some centres opening their catheter laboratories earlier and closing later each day, thereby increasing the number of available working hours.

There was a smaller increase $(10.4 \%)$ in the mean grand total of procedures undertaken in private centres over the same period from 981 in 1992 to 1083 in 1996, and a calculated increase in the mean annual grand total of procedures undertaken per private catheter laboratory of 817 in 1992 and 902 in 1996, and per annual catheter laboratory session of 103 and 120, respectively (table 2). This smaller number of procedures per session in the private sector when compared to the NHS is likely to reflect the more limited demand for private procedures and the ability of the private sector to meet its demands within existing facilities without significant waiting lists.

STAFFING

In NHS centres diagnostic cardiac catheterisation is undertaken by consultant cardiologists, cardiologists in training and, to a lesser extent,

Table 2 Number of catheter laboratories, volume of procedures, and staffing levels in private centres surveyed

\begin{tabular}{|c|c|c|c|c|c|}
\hline & 1992 & 1993 & 1994 & 1995 & 1996 \\
\hline Mean number of catheter laboratories per centre & 1.2 & 1.2 & 1.2 & 1.0 & 1.2 \\
\hline Mean number of adult catheter sessions per week & 9.5 & 13.0 & 11.5 & 13.0 & 9.0 \\
\hline Mean annual number of adult diagnostic angiograms per year & 815 & 764 & 742 & 838 & 909 \\
\hline Mean annual number of adult pacemaker procedures per year & 29 & 32 & 33 & 32 & 37 \\
\hline Mean annual number of adult intervention procedures per year & 137 & 119 & 123 & 138 & 137 \\
\hline $\begin{array}{l}\text { Mean number of consultant cardiologists undertaking diagnostic } \\
\text { catheterisation per centre }\end{array}$ & 9.6 & 12.3 & 14.5 & 16.3 & 17.7 \\
\hline $\begin{array}{l}\text { Mean number of consultant radiolologists undertaking diagnostic } \\
\text { catheterisation per centre }\end{array}$ & 1.7 & 2.3 & 3.2 & 2.3 & 3.3 \\
\hline Mean number of trained PTCA operators (cardiologists) per centre & 8.2 & 12.3 & 11.2 & 10.5 & 6.0 \\
\hline Mean number of trained PTCA operators (radiologists) per centre & 1.0 & 1.6 & 2.5 & 4.5 & 3.5 \\
\hline
\end{tabular}


by radiologists. Very few radiologists are currently being trained to undertake cardiac catheterisation, only five such individuals being reported in 1996. Both the mean number of consultant cardiologists and cardiologists in training undertaking diagnostic catheterisation increased over the five year period, from 4.8 and 3.9 per centre in 1992 , to 7.6 and 6.3 per centre, respectively, in 1996 (table 1). The increase in consultant catheterisers is likely to be caused mainly by an increase in the number of diagnostic catheter sessions allocated in interventional centres to "visiting" district hospital consultants without catheter facilities in their own hospitals. The mean number of trained cardiologists undertaking PTCA also increased over the five year period from 4.8 to 6.7 per centre. This figure consists mostly of consultant staff, both those whose principal employment is within the centre and those undertaking intervention whose principal employment is in a district hospital. However, it also includes some cardiologists in training, mostly those holding the now obsolete "senior registrar" contract rather than the newer "specialist registrar" post, who have completed the required number of procedures to be termed an independent operator. The number of radiologists trained in angioplasty remains small at 0.3 per centre in 1996.

In the private centres cardiac catheterisation and interventional procedures are all undertaken by consultant staff, with the mean number of consultant cardiologists performing diagnostic catheterisation increasing from 9.6 to 17.7 per centre and radiologists from 1.7 to 3.3 over the five year period (table 2). This considerable increase in the mean number of consultant cardiologists undertaking diagnostic catheterisation in the private sector was heavily affected by a single centre with over 25 individuals, and by the small number of private centres returning these data. There was no consistent trend in the number of consultant cardiologists or radiologists undertaking PTCA in private centres.

Since consultant catheterisers and consultant interventionists may undertake procedures in more than one centre, and indeed most will undertake work in at least one NHS and one private hospital, the total number of individuals involved will be significantly less than the figures reported here, which relate to the number of operators undertaking work in each centre.

CORONARY INTERVENTION PROCEDURES

Centres were asked to provide information regarding the number of procedures in each category, and their outcome in terms of angio- graphic success, in-hospital mortality, incidence of periprocedural acute myocardial infarction, and requirement for urgent CABG resulting from an unsatisfactory procedural outcome. Angiographic success relates to the number of vessels attempted, rather than the number of procedures.

All centres undertaking coronary intervention provided the total number of procedures undertaken in their institution for each of the five years reported here. The majority also provided data on outcome; where these data were provided these related to all the relevant procedures undertaken in those institutions for that year. Where percentage rates are reported for the angiographic success and complications of reported procedures, the number of procedures to which the percentage figure refers is given below it in parentheses. Thus, although the percentage rates given do not represent averages derived from every procedure undertaken in the UK, they are derived from all procedures undertaken in the centres that did return data, which in turn was usually a majority of the centres undertaking the procedures. The case mix will obviously differ between centres, but the large number of centres returning data and the high number of procedures from which data were derived does allow a good estimate of national outcome to be made even in the absence of data from some centres.

It was disappointing to see a consistent trend towards fewer centres providing complete data, perhaps reflecting the lack of formally funded audit infrastructure locally, coupled with an almost doubling of the total number of procedures over the five years. Recent developments in the UK, and the subsequent public and political interest generated, make it likely that greater emphasis will be placed on the importance of centres, and individual operators, collecting data in the future. Hopefully sufficient additional resources will be allocated to allow the proper collection of such information.

\section{Centres and activity}

The total number of interventional centres remained almost constant over the five year period at $52-54$, with the majority $(77 \%)$ being NHS hospitals and the minority $(23 \%)$ being private.

All the private centres and $87 \%$ of NHS centres had cardiac surgical facilities on site, those without surgery having arrangements for off site surgical cover locally (table 3 ). Of all the PTCA procedures undertaken in the UK each year, $96 \%$ were performed in the centres with surgery on site in 1992 and this had fallen

Table 3 Total number of interventional centres with cardiac surgical facilities on site and off site

\begin{tabular}{|c|c|c|c|c|c|}
\hline & 1992 & 1993 & 1994 & 1995 & 1996 \\
\hline \multicolumn{6}{|l|}{ On site surgery } \\
\hline Number of centres & 47 & 47 & 47 & 48 & 47 \\
\hline Total annual number of PTCA procedures per centre & 11127 & 12376 & 13802 & 16341 & 19129 \\
\hline Mean annual number of procedures per centre & 237 & 263 & 294 & 340 & 407 \\
\hline \multicolumn{6}{|l|}{ Off site surgery } \\
\hline Number of centres & 5 & 5 & 6 & 6 & 6 \\
\hline Total annual number of PTCA procedures per centre & 448 & 561 & 822 & 1003 & 1382 \\
\hline Mean annual number of procedures per centre & 90 & 112 & 137 & 167 & 230 \\
\hline
\end{tabular}


Table 4 All coronary interventional procedures

\begin{tabular}{llllll}
\hline & 1992 & 1993 & 1994 & 1995 & 1996 \\
\hline Total procedures & 11575 & 12937 & 14624 & 17344 & 20511 \\
Rate per million population & 203 & 227 & 256 & 304 & 360 \\
Total number of centres & 52 & 52 & 53 & 54 & 53 \\
Mean number of procedures/centre & 223 & 244 & 271 & 321 & 380 \\
Angiographic success (\%) & 86 & 89 & 89 & 89 & 90 \\
Myocardial infarction (\%) & $1.4(10294)$ & $1.1(11508)$ & $1.1(12253)$ & $1.4(11365)$ & $1.2(14702)$ \\
CABG (\%) & $2.0(10649)$ & $2.0(11508)$ & $1.8(12598)$ & $1.9(12002)$ & $1.5(15176)$ \\
Mortality (\%) & $0.91(10483)$ & $0.62(11859)$ & $0.72(12598)$ & $0.77(12002)$ & $0.84(15204)$ \\
Range of mortality (\%) & $0-3.5$ & $0-2.7$ & $0-2.7$ & $0-3.4$ & $0-3.2$ \\
\hline
\end{tabular}

Table 5 Single vessel coronary intervention procedures

\begin{tabular}{llllll}
\hline & 1992 & 1993 & 1994 & 1995 & 1996 \\
\hline Total procedures & 8924 & 10046 & 10862 & 9456 & 12605 \\
Number of centres reporting & 48 & 49 & 47 & 34 & 36 \\
Mean number of procedures/centre & 186 & 205 & 231 & 278 & 350 \\
Angiographic success (\%) & 88 & 90 & 89 & 89 & 91 \\
Myocardial infarction (\%) & $1.4(6921)$ & $1.2(9389)$ & $1.1(9474)$ & $1.3(8402)$ & $1.2(11520)$ \\
CABG (\%) & $2.1(8486)$ & $1.8(9808)$ & $1.9(9969)$ & $1.9(9019)$ & $1.4(12239)$ \\
Mortality (\%) & $0.61(7994)$ & $0.57(9438)$ & $0.70(9972)$ & $0.61(8814)$ & $0.74(10872)$ \\
Range of mortality (\%) & $0-3.5$ & $0-3.3$ & $0-3.0$ & $0-2.8$ & $0-3.0$
\end{tabular}

slightly to $93 \%$ by 1996 . The mean number of procedures undertaken in each surgical centre rose from 237 to 407 and in non-surgical centres from 90 to 230, respectively, over the five year period.

The mean number of procedures undertaken annually per NHS centre increased by $85 \%$ over the five year period, from 243 in 1992 to 460 in 1996 . In contrast, over the same period there was no increase in the annual number of procedures undertaken in private hospitals, remaining between 119-137 procedures per centre.

\section{Total PTCA procedures}

Every centre was able to provide the total number of procedures undertaken each year in their institution, resulting in an accurate measurement of national activity. Total coronary intervention procedures increased over the five year period by $77 \%$ to 20511 in 1996, representing an increase in the mean annual number of procedures per centre from 223 in 1992 to 380 in 1996 (table 4). This represents 359 angioplasty procedures per million population in 1996, an increase from 203 per million in 1992 and 88 per million in 1988 (fig 1).

Angiographic success remained fairly constant at around $89 \%$ and mortality rates ranged from $0.62-0.91 \%$, covering a spectrum of clinical conditions. Requirement for urgent

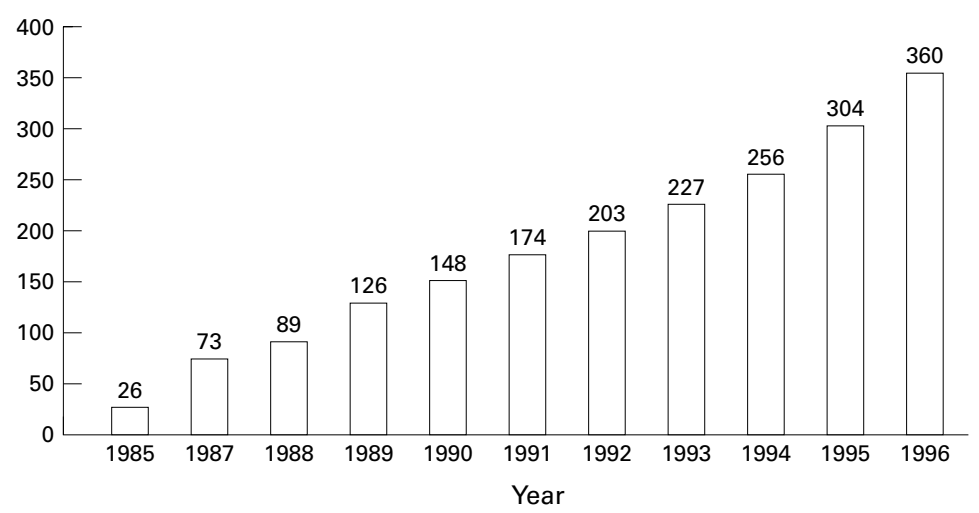

Figure 1 PTCA procedures per million UK population.
CABG was $1.5-2.0 \%$ overall with some suggestion of a slight downward trend over the five year period from $2.0 \%$ in 1992 to $1.5 \%$ in 1996, a trend which was seen in both the single vessel and multivessel subgroups of procedures. One could speculate that this may have been caused by the increased use of intracoronary stenting, especially since most of the downward trend occurred between 1995 and 1996, a period when intracoronary stenting increased from $28 \%$ to $46 \%$ of all procedures.

The number of NHS centres undertaking fewer than 200 procedures per annum, the minimum annual number recommended by the British Cardiac Society and British Cardiovascular Intervention Society, ${ }^{5}$ fell from 16 in 1992 to five in 1996 . Only three of the 12 private centres undertaking PTCA in 1996 performed more than 200 procedures per annum.

Single vessel procedures

The vast majority of angioplasty procedures undertaken were single vessel procedures, remaining fairly constant at $82-85 \%$ of total activity and with the mean number per centre undertaken each year increasing from 186 in 1992 to 350 in 1996 (table 5). Angiographic success was consistently around $90 \%$ and mortality ranged from $0.57-0.74 \%$. The need for urgent CABG showed a possible slight downward trend over the five years from $2.1 \%$ to $1.4 \%$.

\section{Multivessel procedures}

The mean number of multivessel procedures undertaken per centre increased from 40 to 76 over the five years (table 6), but the number undertaken as a proportion of all procedures performed remained remarkably constant at $15-18 \%$. The vast majority of multivessel procedures involved the dilatation of two vessels, and only a few involved more than two, giving a consistent mean number of vessels dilated per multivessel case of around 2.1. Angiographic success rates and requirement for urgent CABG were similar to cases involving single vessel dilatation. Mortality was higher for mul- 
Table 6 Multivessel coronary interventional procedures

\begin{tabular}{llllll}
\hline & 1992 & 1993 & 1994 & 1995 & 1996 \\
\hline Total procedures & 1976 & 2018 & 2014 & 1598 & 2674 \\
Number of centres reporting & 49 & 49 & 47 & 34 & 35 \\
Mean number of procedures/centre & 40 & 41 & 43 & 47 & 76 \\
Mean number of vessels per case & 2.12 & 2.14 & 2.09 & 2.09 & 2.09 \\
Angiographic success (\%) & 88 & 91 & 89 & 90 & 90 \\
Myocardial infarction (\%) & $1.8(1354)$ & $1.9(1572)$ & $1.3(1911)$ & $1.6(1316)$ & $1.1(2461)$ \\
CABG (\%) & $2.1(1620)$ & $2.4(1624)$ & $1.6(1911)$ & $2.2(1474)$ & $1.1(2583)$ \\
Mortality (\%) & $1.07(1496)$ & $0.77(1429)$ & $1.20(1923)$ & $1.52(1316)$ & $1.14(2461)$ \\
Range of mortality (\%) & $0-8.3$ & $0-13.8$ & $0-21.3$ & $0-7.0$ & $0-6.2$ \\
\hline
\end{tabular}

Table 7 Graft coronary interventional procedures

\begin{tabular}{llllll}
\hline & 1992 & 1993 & 1994 & 1995 & 1996 \\
\hline Total procedures & 834 & 1021 & 1107 & 1010 & 1129 \\
Number of centres reporting & 51 & 48 & 41 & 33 & 23 \\
Mean number of procedures/centre & 16 & 21 & 27 & 31 & 49 \\
Angiographic success (\%) & 89 & 90 & 90 & 88 & 91 \\
Myocardial infarction (\%) & $0.8(656)$ & $1.4(994)$ & $1.6(1199)$ & $1.3(927)$ & $1.7(1125)$ \\
CABG (\%) & $0.6(686)$ & $0.6(994)$ & $0.4(1199)$ & $0.4(927)$ & $2.0(1129)$ \\
Mortality (\%) & $1.00(684)$ & $1.50(994)$ & $0.42(1199)$ & $0.64(934)$ & $1.06(1129)$ \\
Range of mortality (\%) & $0-6.1$ & $0-8.3$ & $0-4.5$ & $0-14.3$ & $0-4.7$
\end{tabular}

tivessel than single vessel procedures in each of the five years, with an overall mean mortality over this period for multivessel procedures of $1.14 \%$ compared to $0.65 \%$ for those involving a single vessel. Also, the range of mortalities appeared to be very much higher for multivessel than single vessel procedures, but on closer analysis this was always because of one or two centres in each year having a significantly higher mortality rate. These "higher mortality" centres tended to have undertaken below the mean number of multivessel procedures for that year.

Procedures on coronary bypass grafts

Procedures undertaken on coronary bypass grafts, both venous and arterial, had an angiographic success rate of around $90 \%$ and a rather variable mortality $(0.42-1.5 \%)$ over the five year period, with no consistent trend (table 7). Repeat CABG undertaken urgently for a complication of PTCA is known to carry a much higher mortality than elective CABG and this is likely to be the explanation for the lower referral rate for urgent CABG in this subgroup of patients (approximately 0.5\%) compared to single vessel procedures (approximately $1.8 \%$ ) in the first four years reported. In 1996 the situation was reversed with a referral rate for $\mathrm{CABG}$ in graft procedures increasing from $0.4 \%$ in 1995 to $2.0 \%$ in 1996 , compared to $1.4 \%$ for single vessel procedures in 1996 . Closer analysis showed this trend to have been present in a number of centres, many of which had undertaken considerably more than the mean number of relevant procedures for that year. The mean number of graft procedures per centre increased by $58 \%$ between 1995 and 1996 , possibly associated with a change in case mix, and the number of centres reporting data fell from 33 to 21, which may also have influenced the mean. One can only speculate as to the reasons for this apparent increase in requirement for urgent $\mathrm{CABG}$ in patients undergoing graft angioplasty and await results from subsequent years to determine whether a consistent trend emerges.
Procedures for restenotic lesions

The incidence of angiographic restenosis in clinical trials of balloon angioplasty is reported as being $30-50 \%$ whereas the incidence of "clinical restenosis"-that is, the frequency with which patients return with symptoms following a procedure and require repeat intervention - has always been much lower in clinical practice, possibly closer to $15-20 \%$ in the UK. When one considers procedures involving the insertion of intracoronary stents, trial data suggest an angiographic restenosis of around $10-20 \%$, and in routine clinical practice this may result in repeat intervention in nearer $5-10 \%$ of cases. It is therefore of interest that the mean number of procedures undertaken for restenosis constituted only $11.6 \%$ of the mean number of total angioplasty procedures undertaken per centre in 1992, and that this had fallen slightly to $9.4 \%$ in 1996 , perhaps as a result of the increase in intracoronary stenting (table 8). While underreporting may have contributed to this rate being so low, there is no doubt that the requirement for repeat procedures is considerably lower in routine clinical practice than might be anticipated from randomised clinical trials. This observation is particularly relevant to those assessing the economic implications of angioplasty in the UK because the financial consequences of restenosis, at least in terms of repeat invasive procedures, are considerably less than might be anticipated from the trial data.

Angiographic success was high for procedures involving restenotic lesions and both mortality and requirement for urgent CABG were comparable to or slightly lower than that for single vessel procedures.

\section{Procedures for chronic occlusion}

As expected from experience and clinical trials, the angiographic success for procedures involving chronic occlusions was significantly lower than for other subgroups and over the last few years was consistently around $60-65 \%$. Mortality rates were low for four of the five years reported, averaging $0.21 \%$, but in 1995 was 
Table 8 Interventional procedures for restenosis

\begin{tabular}{llllll}
\hline & 1992 & 1993 & 1994 & 1995 & 1996 \\
\hline Total procedures & 1162 & 1379 & 1282 & 991 & 1059 \\
Number of centres & 45 & 46 & 41 & 32 & 29 \\
Mean number of procedures/centre & 26 & 30 & 31 & 31 & 36 \\
\% of total procedures & 11.6 & 12.3 & 11.4 & 9.6 & 9.4 \\
Angiographic success (\%) & 90 & 92 & 89 & 87 & 89 \\
Myocardial infarction (\%) & $0.4(1095)$ & $0.6(1326)$ & $1.4(1282)$ & $1.1(991)$ & $1.1(1059)$ \\
CABG (\%) & $1.0(1153)$ & $1.6(1326)$ & $2.1(1228)$ & $2.4(991)$ & $1.9(1059)$ \\
Mortality (\%) & $0.28(1062)$ & $0.30(1326)$ & $0.57(1228)$ & $0.55(901)$ & $0.85(1059)$ \\
Range of mortality (\%) & $0-4.3$ & $0-3.7$ & $0-11.1$ & $0-5.9$ & $0-3.0$ \\
\hline
\end{tabular}

Table 9 Interventional procedures for chronic occlusions

\begin{tabular}{llllll}
\hline & 1992 & 1993 & 1994 & 1995 & 1996 \\
\hline Total procedures & 1039 & 1088 & 893 & 1089 & 1226 \\
Number of centres reporting & 50 & 45 & 40 & 32 & 30 \\
Mean number of procedures/centre & 21 & 24 & 22 & 34 & 41 \\
Angiographic success (\%) & 48 & 62 & 65 & 63 & 60 \\
Myocardial infarction (\%) & $0.3(950)$ & $0.4(1058)$ & $0.7(936)$ & $1.4(962)$ & $0.6(1226)$ \\
CABG (\%) & $1.2(950)$ & $0.4(1031)$ & $0.8(878)$ & $0.73(962)$ & $0.61(1152)$ \\
Mortality (\%) & $0.00(950)$ & $0.38(1058)$ & $0.23(881)$ & $1.10(962)$ & $0.26(1152)$ \\
Range of mortality (\%) & 0 & $0-8.3$ & $0-5.0$ & $0-4.3$ & $0-2.6$
\end{tabular}

unexpectedly high at $1.1 \%$ (table 9 ). On closer inspection almost all of the deaths in this year occurred in the two centres undertaking the largest number of procedures on chronic occlusions. One can only speculate why this might have resulted in a higher mortality for these centres, but more complex or higher risk cases may have been undertaken. Whatever the explanation, the rate fell back to a low level by the following year. One conclusion that might be drawn from these data is that although restenotic lesions can carry a low risk, the risk is not zero and can increase to levels comparable to angioplasty for multivessel disease under certain circumstances. Case selection is probably just as relevant when considering PTCA for chronic occlusion as it is for other subgroups of cases.

\section{Procedures for unstable angina}

This is defined as angioplasty undertaken within two weeks of admission to hospital for rest pain. The mean number of angioplasty procedures per centre undertaken for patients with unstable angina increased by $159 \%$ over the five year period, with the majority of this dramatic increase occurring in the last two years. This increase is likely to be a reflection of the evolving experience that procedures can be safely undertaken in this unstable clinical situation and more recently the considerable increase in the use of intracoronary stents. Angiographic success rates were high, around $90 \%$ (table 10), and mortality rates and requirement for urgent $\mathrm{CABG}$ only slightly higher than the mean for all procedures.
Procedures for acute myocardial infarction

This is defined as angioplasty undertaken within two weeks of proven acute myocardial infarction. The number of procedures undertaken for acute myocardial infarction remained a small percentage of total activity throughout the five year period, with the mean number of procedures per centre rising only to 15 per centre in 1996 (table 11). Given that by 1996 a significant amount of encouraging data had been reported relating to "primary angioplasty" for acute myocardial infarction, this low level of activity may seem surprising. However, for centres to provide an interventional service for acute infarction requires a significant increase in departmental infrastructure and often changes in working practices, all of which require additional funds for the service to be developed to anything approaching clinical need. These factors probably explain the slow growth of this interventional area to date. More rapid growth may occur over the next five years.

As might be anticipated the mortality rate for intervention undertaken in the context of evolving infarction is significantly higher than the mean for all procedures, and was three to seven times as high as for procedures undertaken on patients with unstable angina, with no consistent downward trend over time. Nevertheless, although the patient group reported is obviously a selected one, a mortality rate of around $4-7 \%$ compares favourably to general experience of unselected admissions to hospital with acute infarction where the overall mortality is usually closer to $15 \%$. This favourable comparison was

Table 10 Coronary interventional procedures for unstable angina

\begin{tabular}{llllll}
\hline & 1992 & 1993 & 1994 & 1995 & 1996 \\
\hline Total procedures & 2200 & 2703 & 2704 & 2698 & 3682 \\
Number of centres reporting & 48 & 45 & 41 & 33 & 31 \\
Mean number of procedures/centre & 46 & 60 & 66 & 82 & 119 \\
Angiographic success (\%) & 90 & 90 & 90 & 90 & 91 \\
Myocardial infarction (\%) & $1.8(2100)$ & $1.7(2503)$ & $1.7(2604)$ & $2.1(2588)$ & $1.9(3189)$ \\
CABG (\%) & $2.7(2100)$ & $2.7(2503)$ & $2.2(2604)$ & $2.4(2578)$ & $1.6(3181)$ \\
Mortality (\%) & $0.86(2100)$ & $0.88(2503)$ & $1.19(2604)$ & $0.83(2540)$ & $1.25(3189)$ \\
Range of mortality (\%) & $0-16.7$ & $0-11.1$ & $0-12.5$ & $0-3.4$ & $0-4.2$ \\
\hline
\end{tabular}


Table 11 Coronary interventional procedures for acute myocardial infarction

\begin{tabular}{llllll}
\hline & 1992 & 1993 & 1994 & 1995 & 1996 \\
\hline Total procedures & 191 & 231 & 238 & 285 & 457 \\
Number of centres reporting & 51 & 46 & 38 & 32 & 31 \\
Mean number of procedures/centre & 4 & 5 & 6 & 9 & 15 \\
Angiographic success (\%) & 76 & 85 & 84 & 84 & 85 \\
CABG (\%) & $2.3(172)$ & $5.2(231)$ & $2.6(229)$ & $6.1(246)$ & $3.2(376)$ \\
Mortality (\%) & $5.81(172)$ & $4.76(231)$ & $3.93(229)$ & $4.88(246)$ & $6.63(377)$ \\
Range of mortality (\%) & $0-50.0$ & $0-30.8$ & $0-100$ & $0-33.4$ & $0-28.6$ \\
\hline
\end{tabular}

Table 12 Total coronary stent procedures

\begin{tabular}{lcccrc}
\hline & 1992 & 1993 & 1994 & 1995 & 1996 \\
\hline Total stent procedures & 319 & 660 & 1937 & 4573 & 9165 \\
Number of centres reporting & 50 & 53 & 54 & 51 & 51 \\
$\begin{array}{l}\text { Mean number of stent procedures/centre } \\
\text { \% of total procedures involving stent insertion }\end{array}$ & 6 & 12 & 36 & 90 & 180 \\
& 2.7 & 5.6 & 13.5 & 27.6 & 45.9 \\
\hline
\end{tabular}

Table 13 Mean volume of other procedures (cases per centre per year)

\begin{tabular}{llllll}
\hline & 1992 & 1993 & 1994 & 1995 & 1996 \\
\hline Directional atherectomy & 2.1 & 2.1 & 1.4 & 1.6 & 1.0 \\
Rotational atherectomy & 0.6 & 0.7 & 1.7 & 0.9 & 4.1 \\
Mitral valvuloplasty & 5.2 & 7.7 & 7.6 & 9.6 & 8.0 \\
Aortic valvuloplasty & 0.4 & 0.2 & 0.6 & 0.5 & 0.3 \\
Dilatation of aortic coarct or recoarctation & 0.4 & 0.4 & 0.2 & 0.3 & 0.5 \\
\hline
\end{tabular}

also seen in the Exeter primary angioplasty pilot study, a UK trial where primary angioplasty was undertaken on a cohort of sequential unselected patients being admitted with acute myocardial infarction to a district general hospital. ${ }^{6}$

\section{Stent procedures}

The most dramatic change over the five year period was the increase in the number of procedures involving the insertion of intracoronary stents, reflecting similar developments elsewhere in Europe and in the USA. Data on the total number of stent procedures were obtained from all interventional centres for each of the five years, giving an accurate indication of national activity. The number of stent procedures increased from only 319 in 1992 to 9165 in 1996, a rise of $2773 \%$ (table 12). In 1992 only $2.7 \%$ of all procedures involved the insertion of a stent, whereas this figure had increased to $45.9 \%$ by 1996 . In this last year centres were asked how many stents they had used, in addition to the number of procedures involved; from the 26 centres supplying data it was calculated that a mean of 1.26 stents were inserted per procedure. If the remaining centres had similar stent use the calculated total for stent sales in the UK in 1996 would have been 11548 . Data relating to results and outcome following stent procedures were not specifically requested over this five year period and they appear within the other subgroups listed above.

\section{Volume of other procedures}

Only information relating to the total number of procedures undertaken was sought about coronary procedures involving devices other than balloons and stents, and other interventional cardiovascular procedures. The mean number of procedures per centre are shown in table 13, from which it can be seen that levels of activity in these areas are low. This is either because of a low prevalence of the relevant condition, as is the case with mitral stenosis suitable for mitral valvuloplasty, or a relatively low uptake of the devices compared to other countries, as in the case of rotational and directional atherectomy. Having experienced a surge in interest in the late $1980 \mathrm{~s}$, aortic valvuloplasty has since been undertaken only rarely.

\section{Conclusions}

Data were collected from all centres undertaking percutaneous cardiac interventional procedures in the UK between 1992 and 1996. The principal observations were as follows:

(1) Within NHS interventional centres the mean number of catheter laboratories increased from 1.7 in 1992 to 1.9 in 1996, and the total throughput of these NHS laboratories (diagnostic catheters, pacemakers, and intervention procedures) rose by $42 \%$. There was a smaller increase in throughput $(10.4 \%)$ in private centres and no increase in the number of their catheter laboratories.

(2) Over the five years, the mean number of consultant cardiologists and cardiologists in training who undertook diagnostic catheterisation in NHS interventional centres both increased by about $60 \%$.

(3) The number of centres undertaking coronary intervention procedures remained almost constant over the five year period (range 52-54). In 1996 a total of $89 \%$ of all centres had cardiac surgical facilities and these "surgical" centres undertook $93 \%$ of all the angioplasty procedures in the UK.

(4) The number of trained angioplasty operators in NHS centres increased by $40 \%$ over the five year period.

(5) Total coronary intervention procedures increased by $77 \%$ from 11575 in 1992 to 20511 in 1996, which represents a rise from 203 to 360 procedures per million UK population, respectively. 
(6) The number of NHS interventional centres undertaking fewer than 200 procedures per year fell from 16 in 1992 to five in 1996.

(7) The number of procedures undertaken for unstable angina increased $159 \%$ over the five years, but relatively few procedures were undertaken for acute myocardial infarction, even in 1996.

(8) There was a very large increase $(2773 \%)$ in the number of intervention procedures involving the insertion of an intracoronary stent, from 319 in 1992 to 9165 in 1996. A mean of 1.26 stents were inserted per procedure.

(9) When all coronary intervention procedures for each year were grouped together, angiographic success was 86$90 \%$, mortality was $0.6-0.9 \%$, and $1.5-$ $2.0 \%$ of cases required urgent CABG, over the five year period.

(10) All centres provided data on the number of procedures undertaken for each of the five years, but $17 \%$ of NHS centres and $67 \%$ of private hospitals were unable to provide data on mortality or other outcome measures in 1996.

\section{Appendix 1}

The British Cardiovascular Intervention Society gratefully acknowledges the cooperation and help of the following hospitals, without which this audit process could not have been conducted. A few centres have either closed, amalgamated with others or stopped undertaking angioplasty during the five year period reported. Hence the number of hospitals recorded here is greater than the total number of centres returning data in any single year reported.

NHS HOSPITALS

Aberdeen Royal Infirmary; Airedale Hospital; City Hospital, Belfast; Royal Victoria Hospital, Belfast; Dudley Road Hospital, Birmingham; Heartlands Hospital, Birmingham; Queen Elizabeth Hospital, Birmingham; Royal Victoria Hospital, Blackpool; Bristol Royal
Infirmary; Papworth Hospital, Cambridge; Cardiff University Hospital; Walsgrave Hospital, Coventry; Western General Hospital, Edinburgh; Royal Devon and Exeter Hospital; Royal Infirmary, Edinburgh; Royal Infirmary, Glasgow; Western Hospital, Glasgow; Royal Infirmary, Hull; Leeds General Infirmary; Killingbeck Hospital, Leeds; Groby Road and Glenfield Hospitals, Leicester; Cardiothoracic Centre, Liverpool; Guys Hospital, London; Brook Hospital, London; Hammersmith Hospital, London; Harefield Hospital, London; Kings College Hospital, London; London Chest Hospital, London; Middlesex Hospital, London; Royal Brompton Hospital, London; Royal Free Hospital, London; Royal London Hospital, London; St Bartholomew's Hospital, London; St George's Hospital, London; St Thomas' Hospital, London; St Mary's Hospital, London; Westminster Hospital, London; Manchester Royal Infirmary; Wythenshaw Hospital, Manchester; Freeman Hospital, Newcastle; City and University Hospitals, Nottingham; John Radcliffe Hospital, Oxford; Northern Hospital, Sheffield; Southampton University Hospitals; South Cleveland Hospital, Middlesbrough; Stoke-on-Trent Hospital.

PRIVATE HOSPITALS

BUPA Cambridge Lea, Cambridge; Ross Hall Hospital, Glasgow; BUPA Hospital, Leeds; BUPA Hospital, Leicester; London Bridge Hospital, London; Cromwell Hospital, London; Harley St Clinic, London; London Independent Hospital, London; St Anthony's Hospital, London; Wellington Hospital, London; Alexandra Hospital, Manchester; BMI Priory Hospital, Birmingham.

1 Hubner PJB, for Council of the British Cardiovascular Intervention Society. Cardiac interventional procedures in the United Kingdom during 1988. Br Heart $\mathcal{F} 1990 ; 64: 36-$ 7.

2 Hubner PJB, for Council of the British Cardiovascular Intervention Society. Cardiac interventional procedures in the United Kingdom in 1989. Br Heart f 1991; 66:469-71.

3 Hubner PJB, for Council of the British Cardiovascular Intervention Society. Cardiac intervention procedures in the United Kingdom in 1990. Br Heart F 1992;68:434-6.

4 Council of the British Cardiovascular Intervention Society. Cardiac intervention procedures in the United Kingdom in Cardiac intervention procedures in

5 Parker DJ, Gray HH, Balcon R, et al. Planning for coronary angioplasty: guidelines for training and continuing compeangioplasty: guidelines for trai
tence. Heart 1996;75:419-25.

6 Smith LDR, Dean JW. Primary angioplasty in the district general hospital: interim analysis of the Exeter primary angioplasty pilot study [abstract]. Heart 1997;77(suppl 1):P46 